\title{
Solvent-sensitive Nanospheres Prepared by the Self-organization of Polymerizing Hydrophilic Graft Chain Copolymers
}

\author{
Ming-qing Chen, ${ }^{1, \dagger}$ Kun Zhang, ${ }^{1}$ Tatsuo KAneKo, ${ }^{2}$ Xiao-ya Liu, ${ }^{1}$ \\ Jie CAI, ${ }^{1}$ and Mitsuru AKASHI ${ }^{1,2, \dagger}$ \\ ${ }^{1}$ School of Chemical and Material Engineering, Southern Yangtze University, Wuxi 214036, P. R. China \\ ${ }^{2}$ Department of Molecular Chemistry, Graduate School of Engineering, Osaka University, \\ 2-1 Yamadaoka, Suita 565-0871, Japan
}

(Received September 21, 2004; Accepted November 8, 2004; Published February 15, 2005)

\begin{abstract}
Uniform nanospheres were prepared by dispersion copolymerization of $n$-butyl methacrylate (BMA) with poly(ethylene glycol) (PEG) macromonomers with $p$-vinylbenzyl end groups in various ethanol/water media. Particle size effects were investigated as a function of macromonomer molecular weight, solvent composition, and initial monomer and initiator concentrations. Hydrodynamic nanoparticle radii $\left(R_{\mathrm{h}}\right)$ followed the exponential law of initial initiator, St-PEG macromonomer and BMA concentrations. When reaction conditions were properly chosen, nanosphere particle size was controlled from $c a$. 40 to $750 \mathrm{~nm}$ in diameter. It was found that nanospheres swelled in ethanol/water dispersion media and the degree of swelling responded sensitively to solvent composition changes and reached a maximum at ethanol contents of 40-50 vol \%. [DOI 10.1295/polymj.37.118]

KEY WORDS n-Butyl Methacrylate / Poly(ethylene glycol) Macromonomer / Nanospheres / Dispersion Polymerization / Solvent Sensitivity /
\end{abstract}

Motivated by wide potential nanosphere applicability in the biomedical field, information industries, microelectronics, etc. in recent years many researchers have actively prepared nanospheres. ${ }^{1-4}$ Dispersion polymerization was widely employed as a very useful tool in preparing various nanospheres. In recent years, we have also prepared nano-sized uniform polymeric spheres via free radical dispersion copolymerization of hydrophobic monomers and hydrophilic macromonomers in polar solvents. To obtain nanospheres with different surface functionalities, functional macromonomers such as poly( $N$-isopropylacrylamide $)$ (PNIPAAm) ${ }^{5}$ poly(ethylene glycol) (PEG),${ }^{6-8}$ poly(methacrylic acid) (PMA), ${ }^{9}$ poly $(N$-vinylacetoamide) and their derivatives have been successfully used. ${ }^{10}$ It is widely accepted that the introduction of PEG chains onto nanosphere surfaces is very significant. Materials comprised of PEG on their surfaces are potentially useful in applications requiring contact with biological materials such as cells. ${ }^{11}$ Delgado et al. ${ }^{12}$ analyzed the usefulness and properties of PEG-linked proteins, and Mabrouk ${ }^{13}$ studied the effect of pegylation on the structure and function of proteins. In our studies on core-corona type nanospheres with PEG corona chains, the core was mainly composed of polystyrene (PSt). The PSt core was quite stable and did not change in size or shape with a changing environment. An environment-responsive core may lead to the development of a new class of nanoparticles. Poly(n-butyl methacrylate) (PBMA) under bulk conditions has glass transition temperatures much lower than PSt and is soluble in hot ethanol. These properties may create sensitivity to changes attributed to solvent environments. In the micellar nanoparticles composed of the block copolymers, the significance of the particles changing their size responding to an environmental change have been pointed out in terms of the developing of drug controlled incorporation. ${ }^{14,15}$ Although PBMA has been widely used as a core material in the nanoparticle preparation, ${ }^{16}$ there is no paper referencing core solvent-sensitivity of the core-corona type nanoparticles composed of the graft copolymers which are easily prepared by a one-step radical polymerization using a macromonomer.

In this article, we used free radical dispersion copolymerization of hydrophobic BMA and hydrophilic PEG macromonomers to prepare PBMA- $g$-PEG polymeric nanospheres in ethanol/water mixed solvents, and investigated reaction parameter effects on nanosphere formation. Dispersion medium effects as a function of nanosphere particle size were also investigated.

\section{EXPERIMENTAL}

\section{Materials}

$2,2^{\prime}$-Azobis(isobutyronitrile) (AIBN) was recrystallized from excess ethanol and dried in vacuo. Sodium hydride ( $\mathrm{NaH}$ ) (Nacalai, Kyoto, Japan) was used as received. Solvents such as tetrahydrofuran (THF), etha-

${ }^{\dagger}$ To whom correspondence should be addressed (E-mail: akashi@chem.eng.osaka-u.ac.jp, mqchen@sytu.edu.cn). 
nol, acetone, and diethyl ether were purified in usual ways prior to use. Water was purified by distillation. p-Chloromethylstyrene (CMSt) and PEG with hydroxyl terminal groups (PEG2000 and PEG5000) were supplied by Nippon Oil and Fats Co. (Tokyo, Japan), and were used as received. n-Butyl methacrylate (BMA) (SCR, Shanghai, China) was distilled prior to use.

\section{Preparation of St-PEG Macromonomers}

Polymerizable styrene groups were attached to PEG termini as follows. THF solution $(120 \mathrm{~mL})$ of PEG with a hydroxyl terminal group was stirred with 1.5fold excesses (by an equimolecular amount based on oligomers) of $\mathrm{NaH}$ at $40^{\circ} \mathrm{C}$ for $2 \mathrm{~h}$. After 4-fold excesses of CMSt were added, the reaction solution was maintained at $34{ }^{\circ} \mathrm{C}$ for $24 \mathrm{~h}$. Reaction solutions were poured from THF into acetone and precipitated $\mathrm{NaCl}$ was removed by filtration. The filtrate was precipitated into diethyl ether and purified by re-precipitation (three times). The products (Styrene-terminated PEG; St-PEG macromonomers) were dried in vacuo at room temperature. The molecular weight of St-PEG macromonomers was measured by gel permeation chromatography (GPC) (molecular weight: St-PEG2000 = 2240 and St-PEG5000 = 5015). The degree of St-termination was quantitated by ${ }^{1} \mathrm{H}$ NMR from peak intensity ratios of vinyl to methylene polymer chain protons, showing that double bond contents were 91 and 93\% for St-PEG2000 and St-PEG5000, respectively.

\section{Nanosphere Preparation}

Polymerization was performed as follows: The required amounts of St-PEG macromonomer and BMA were added to a certain amount of initiator and solvent within a polymerization tube. The tube was purged with nitrogen for $10 \mathrm{~min}$, and polymerizations were commenced by immersing the tube in a shaking water bath $(165 \mathrm{rpm}) \mathrm{kept}$ at $60^{\circ} \mathrm{C}$ for $1-$ $24 \mathrm{~h}$. In the polymerization process, the homogeneous solution became translucent, implying nanoparticle preparation. The resulting solution was dialyzed in distilled water using a cellulose dialyzer (cutoff mass: $14,000 \mathrm{Da}$ ) for $3 \mathrm{~d}$ to remove unreacted monomers. The yields calculated from weight ratios of dried sample to total "in fed" monomers were beyond $85 \%$.

\section{Measurement}

Particle sizes were measured using dynamic light scattering (DLS) at $25^{\circ} \mathrm{C}$. Nanosphere hydrodynamic radii $\left(R_{\mathrm{h}}\right)$ were determined by a modified Laser Light Scattering (LLS) spectrometer (ALV/SP-125) equipped with a multi- $\tau$ digital time correlator (ALV$5000 \mathrm{E}$ ) and a He-Ne laser (JDS Uniphase 1100, out- put power $=25 \mathrm{~mW}$ at $\lambda_{0}=632 \mathrm{~nm}$ ). The dispersion medium was water. $R_{\mathrm{h}}$ and its distribution were obtained by a COTIN program. The measurement angle was fixed at $80^{\circ}$.

Number-average molecular weights $\left(M_{\mathrm{n}}\right)$ were measured by gel permeation chromatography (GPC) (Agilent 1100 Series) using PSt standard. $N, N$-Dimethylformamide (DMF) was used as eluent at a flow rate of $0.6 \mathrm{~mL} / \mathrm{min}$ at $35^{\circ} \mathrm{C}$.

${ }^{1} \mathrm{H}$ NMR spectra were measured on a Bruker DMX500 $(500 \mathrm{MHz})$ in dimethylsulfoxide- $d_{6}$. Product morphologies were observed by transmission electron microscopy (TEM, Hitachi H-7010A). The samples were prepared as follows: aqueous dispersion of nanospheres were cast onto a copper mesh on a glass seat and dried at room temperature.

\section{RESULTS AND DISCUSSION}

\section{Nanosphere Preparation}

When a macromonomer is soluble in a water/ethanol solvent, free radical copolymerization of the macromonomer with a solvatophobic monomer in the solvent may yield nanospheres with solvatophobic cores and solvatophilic graft surface chains (corona). In the present work, PBMA- $g$-PEG chains were prepared in water/ethanol solvents. Figure 1a shows a TEM image of dried product from dispersed polymerization of BMA $(5 \mathrm{mmol})$ and St-PEG $(0.0 .033 \mathrm{mmol})$ in the presence of AIBN ( $1 \mathrm{~mol} \%$ to total monomer) in ethanol/water $(\mathrm{v} / \mathrm{v}, 7 / 3,5 \mathrm{~mL})$, following purification, at $60^{\circ} \mathrm{C}$, indicating the successful formation of uniform spherical nanoparticles with $130 \pm 10 \mathrm{~nm}$ diameters. Figure $1 \mathrm{~b}$ shows the result of dynamic light scattering (DLS) measurements of nanosphere dispersion, indicating a narrow distribution in size and $R_{\mathrm{h}}$ $(130 \pm 10 \mathrm{~nm})$ and in good agreement with those of TEM. In other polymerization conditions, the formation of various-sized nanospheres was confirmed by TEM. In the following studies, nanosphere sizes were measured by DLS.

Figure 2 shows the effect of polymerization time on conversion, the $R_{\mathrm{h}}$ of resulting nanospheres, and the molecular weight of graft copolymers. The particle size and the molecular weight increased with polymerization. The curves are nearly linear at the early stage of polymerization and then level off after $6 \mathrm{~h}$. Similar results have been obtained in the polymerization of poly(methacrylic acid) (PMAA) macromonomers with methyl methacrylate (MMA). ${ }^{17,18}$ As Winnik et al. ${ }^{18}$ described in dispersion polymerization, (1) the monomer, macromonomer, and initiator completely dissolve in solvent before polymerization; and (2) linear oligomers and polymers and graft co-oligomers and copolymers are produced early in the continuous 
(a)

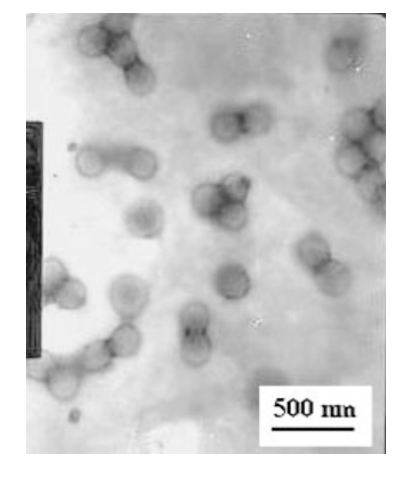

(b)

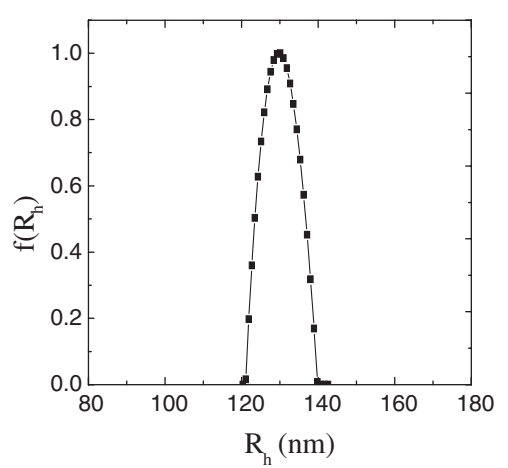

Figure 1. (a) TEM image and (b) $R_{\mathrm{h}}$ distribution of microspheres obtained by dispersion copolymerization of BMA (5 mmol) and StPEG $(0.033 \mathrm{mmol})$ in the presence of $\mathrm{AIBN}\left(1 \mathrm{~mol} \%\right.$ to total monomer) in ethanol/water $(\mathrm{v} / \mathrm{v}, 7 / 3,5 \mathrm{~mL})$ at $60{ }^{\circ} \mathrm{C}$.

(a)

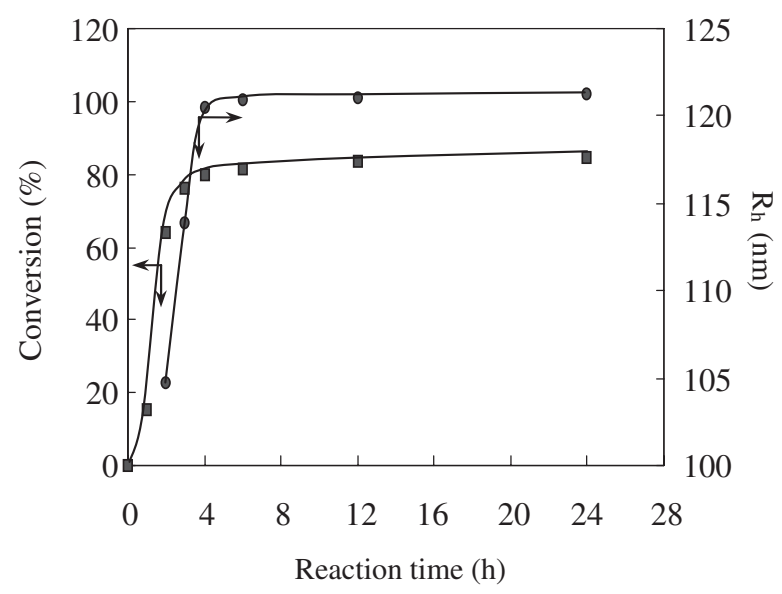

(b)

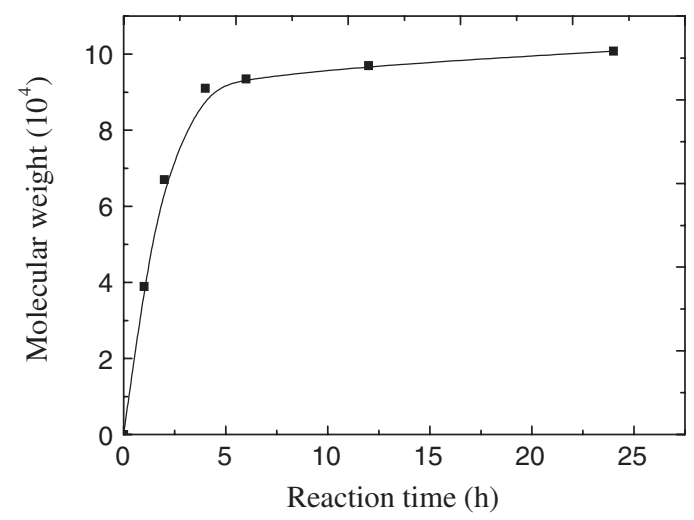

Figure 2. (a) Time-conversion curve ( $\boldsymbol{\square})$ and $R_{\mathrm{h}}$ of resulting nanospheres (-), (b) the effect of reaction time on the copolymer molecular weight in the dispersion copolymerization of BMA $(4.6 \mathrm{mmol})$ and St-PEG $(0.05 \mathrm{mmol})$ in the presence of AIBN $(1 \mathrm{~mol} \%$ to total monomer) in ethanol/water $(\mathrm{v} / \mathrm{v}, 7 / 3,5 \mathrm{~mL})$ at $60^{\circ} \mathrm{C}$. polymerization phase. The molecular weight and composition of these polymers determines their solubilities. According to Fitch and Tsai's homogeneous nucleation theory, ${ }^{19}$ (3) aggregative and coagulative nucleation will occur when the molecular weight of polymers exceeds a critical value. Particle coagulation occurs until sterically stabilized particles form. Based on experimental results and these theories, we speculate the process of nanosphere formation as shown below. No particle having water-dispersion stability was obtained until $2 \mathrm{~h}$ although DLS measurement was allowed under the vigorous agitation. In this stage, it is considered that sufficient PEG chains lack on the particle surface for colloidal stability since the previous studies demonstrated that the hydrophobic methacrylate esters have much higher reactivity than the hydrophilic macromonomers. ${ }^{20,21}$ After $2 \mathrm{~h}$ polymerization, the conversion further increased and copolymers obtained sufficient PEG graft chains to provide stability to the resulting nanospheres. The nanospheres grew in the continuous phase by oligomer diffusive capture and small particle coagulation (nuclei or precursors) as well as by copolymerization with captured monomers that partition into two phases of solvatophobic domain and solution. After $6 \mathrm{~h}$ nanosphere conversion and $R_{\mathrm{h}}$ showed little change regardless of an increase in reaction time. Final nanosphere $R_{\mathrm{h}}$ and molecular weights were $121 \mathrm{~nm}$ and $1.0 \times 10^{5}$, respectively.

In the present experiments, reaction parameter effects on nanospheres were investigated using StPEG macromonomers. Nanospheres with $121 \mathrm{~nm}$ in $R_{\mathrm{h}}$ can be obtained under the following polymerization conditions: $n$-BMA $(4.6 \mathrm{mmol})$, St-PEG (0.05 mmol), initiator ( $1 \mathrm{~mol} \%$ to total monomer), solvent $(5.0 \mathrm{~mL}$, ethanol/water; $7 / 3, \mathrm{v} / \mathrm{v})$, reaction temperature $\left(60^{\circ} \mathrm{C}\right)$ and reaction time $(24 \mathrm{~h})$. These conditions were used as standard polymerization conditions. 


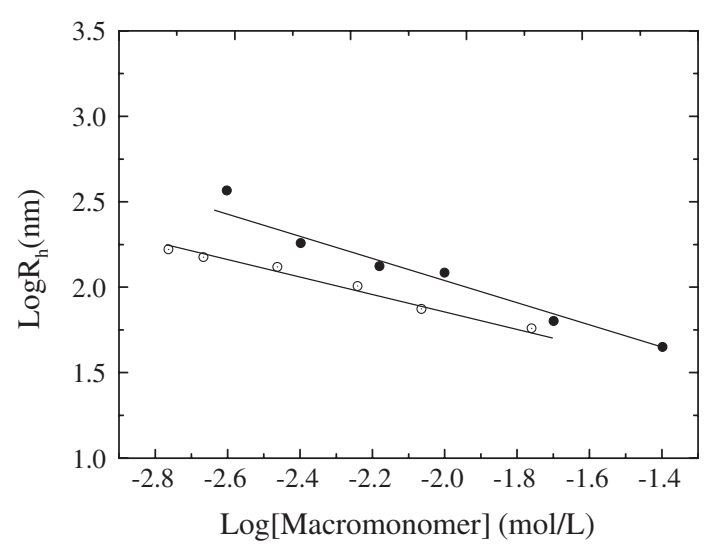

Figure 3. Double-logarithmic plots of nanosphere radii $\left(R_{\mathrm{h}}\right)$ vs. [macromonomer]. (๑) St-PEG2000 (O) St-PEG5000, [BMA] $=0.93 \mathrm{~mol} / \mathrm{L}$ and $T=60^{\circ} \mathrm{C}$ The solvent is ethanol containing $30 \mathrm{vol} \%$ water.

\section{Macromonomer Concentration Effects}

Figure 3 shows a $\log -\log$ plot of nanospheres $R_{\mathrm{h}}$ against the concentration of PEG macromonomers. $R_{\mathrm{h}}$ decreased with a concentration increase in macromonomers when kept the BMA monomer concentration as a constant in the feed. Particle size was dependent on stabilizer concentrations according to literature on systems for non-aqueous dispersion polymerization. ${ }^{22}$ In the present study, the amphiphilic StPEG macromonomers behave as both co-monomers and stabilizers which may affect $R_{\mathrm{h}}$. Although PEG macromonomer showed a critical micelle concentration $(\mathrm{cmc})$ in an aqueous solution ${ }^{23}$ we will not be concerned with $\mathrm{cmc}$ whose effects will be presented in a future study.

According to the literature, particle size may be dependent on the extent of nuclear aggregation occurring by adsorption of the macromonomer whose diffusion was influenced by continuous phase viscosity. ${ }^{24}$ Increasing the macromonomer concentration can increase both the adsorbed macromonomer and continuous phase viscosity. Then, the presence of increased macromonomers can more effectively stabilize particle surfaces and yield smaller nanospheres with larger specific surface areas.

Another important feature, shown in this figure, is the dependence of PEG chain lengths on final particle size. When a macromonomer with a longer chain was used, smaller particles were obtained at equivalent macromonomer concentrations. Two main reasons can arise: 1) the solution viscosity increases with increasing macromonomer molecular weight, which interferes in the growth of aggregating or coalescent particles; and 2) longer PEG chains can occupy larger surface areas, creating smaller particles. The number of PEG chains on the particle surfaces, $n$, and the surface area occupied by each PEG macromonomer,
Table I. The number of grafted PEG chains on a particle surface, $n$, and the surface area occupied by one PEG chain, $s$, in the nanoparticles of Pn-BMA-g-PEG ${ }^{\mathrm{a}}$

\begin{tabular}{ccccccc}
\hline $\begin{array}{c}\text { PEG2000 } \\
(\mathrm{mmol} / \mathrm{L})^{\mathrm{b}}\end{array}$ & $n \times 10^{-4}$ & $\begin{array}{c}s \\
\left(\mathrm{~nm}^{2}\right)\end{array}$ & $\begin{array}{c}\text { PEG5000 } \\
(\mathrm{mmol} / \mathrm{L})^{\mathrm{b}}\end{array}$ & $n \times 10^{-4}$ & $\begin{array}{c}s \\
\left(\mathrm{~nm}^{2}\right)\end{array}$ \\
\hline 40 & 6.8 & 0.37 & & 17.4 & 6.9 & 0.58 \\
20 & 9.8 & 0.52 & & 8.6 & 7.9 & 0.89 \\
10 & 33 & 0.55 & & 5.7 & 13 & 0.99 \\
6.6 & 28 & 0.77 & 3.4 & 17 & 1.28 \\
4 & 44 & 0.92 & 2.2 & 16 & 1.72 \\
2.5 & 230 & 0.73 & & 1.7 & 17 & 2.02 \\
\hline
\end{tabular}

${ }^{\mathrm{a}} R_{\mathrm{h}}$ values are plotted in Figure 3 and $n$-BMA concentration is $0.93 \mathrm{M}$. ${ }^{\mathrm{b}} \mathrm{PEG} 2000$ and PEG5000 refers to styrene-terminated poly(ethylene glycol)s with molecular weights of 2240 and 5015 , respectively.

$s$, are important to explain the effects of macromonomers on the colloid stability of nanospheres. We roughly calculated $n$ and $s$ values according with the method shown in our previous report and summarized in Table I. ${ }^{25}$ It was found that $n$ in PEG2000 is bigger than that in PEG5000, suggesting that the colloid-stabilizing effect of PEG5000 was higher than PEG2000. The higher molecular weight may be more efficient to give the hydrophilicity the particle surface.

From Figure 3, the following scaling behavior is obtained, irrespective of macromonomer: for StPEG2000

$$
R_{\mathrm{h}}=K[\text { Macromonomer }]_{0}-0.65 \pm 0.03
$$

for St-PEG5000

$$
R_{\mathrm{h}}=K[\text { Macromonomer }]_{0}-0.51 \pm 0.02
$$

Paine ${ }^{25}$ predicted an exponent of -0.5 for the stabilizer concentration dependence on nanoparticle size when operating under a grafting stabilization mechanism. The exponent for St-PEG2000 is larger than the theoretical value, indicating that the particle size changes more sensitively to the macromonomer concentration change. However, it is noted that the StPEG5000 exponent agrees with the theoretical value. In addition, we previously reported two exponents, -0.53 and -0.69 , for dispersion copolymerization of MMA-terminated PEG and St-PEG macromonomers with St, respectively. ${ }^{6}$ Although it is quite difficult to explain that St-PEG2000 gave a larger value, we can guess that the conformation change in the small PEG chains sensitive to their surface density on the hydrophilicity of the nanoparticle surface may affect the exponent value.

At low and high macromonomer concentrations we obtained $R_{\mathrm{h}} \sim n_{\mathrm{EO}}^{-0.68}$ and $R_{\mathrm{h}} \sim n_{\mathrm{EO}}^{-0.37}$, respectively. These results indicate that macromonomer ability to stabilize nanospheres depends upon the concentration as well as the length of ethyleneoxide macromonomer 


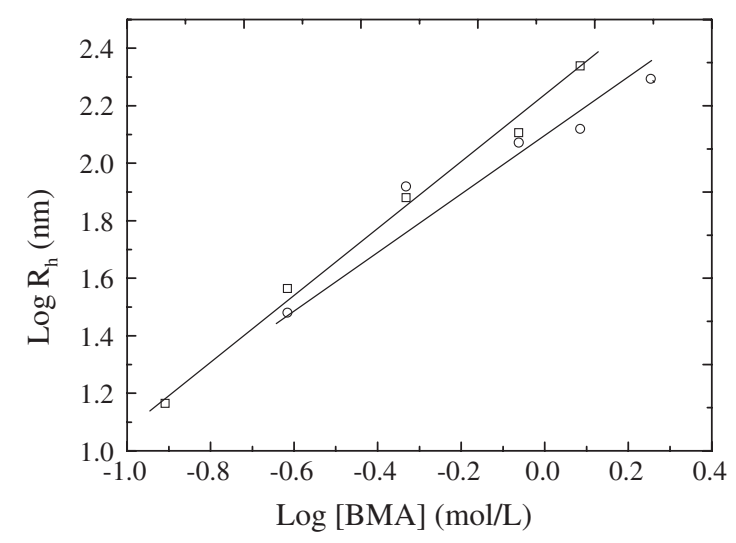

Figure 4. Double-logarithmic plots of nanosphere radii $\left(R_{\mathrm{h}}\right)$ $v s$. [BMA], ( $\square) n$-BMA (○) $t$-BMA $[\mathrm{St}-\mathrm{PEG}]=10 \mathrm{mmol} / \mathrm{L}$, $[\mathrm{I}]=1 \mathrm{~mol} \%[\mathrm{BMA}]$ and $T=60^{\circ} \mathrm{C}$ The solvent is ethanol containing $30 \mathrm{vol} \%$ water.

chains.

\section{Initial BMA Concentration Effects}

Initial monomer concentrations have a significant influence on final particle size. ${ }^{18,26,27}$ Figure 4 shows the initial BMA concentration effect on particle radius. $R_{\mathrm{h}}$ increased significantly from 15 to $218 \mathrm{~nm}$ with increasing initial BMA concentration from $0.12 \mathrm{~mol} /$ $\mathrm{L}$ to $1.8 \mathrm{~mol} / \mathrm{L}$, at a constant St-PEG macromonomer amount. $\mathrm{Wu}^{25}$ mentioned the surface area occupied by PEG macromonomer is nearly a constant; that is to say, the area of the surface stabilized by a given amount of PEG macromonomer is nearly constant. On the other hand, an increased BMA concentration can result in an increase in total core volume since PBMA forms nanosphere cores. Thus, larger particles with smaller specific surface areas were formed when the initial BMA concentration was increased. In other words, the major effect of monomer concentration refers to the initial solvation ability of the reaction medium. At higher initial concentrations, increased PBMA permit more coalescence and generate larger particles.

We also investigated butyl group structure effects ( $t$-BMA and $n$-BMA) on particle size. It is noticeable that $R_{\mathrm{h}}$ of nanospheres formed by copolymerization of $t$-BMA and St-PEG are smaller than those formed by copolymerization of $n$-BMA and St-PEG. The possible explanation is that the polymer chain formed by copolymerization with $t$-BMA and St-PEG is more solvatophobic, easier to shrink and forms denser cores compared to $n$-BMA and St-PEG polymer chains. Denser packing of bulkier $t$-butyl group of $t$-BMA seems more difficult than $n$-BMA. However, we proposed the effect of the glass transition temperature, $T_{\mathrm{g}}$, representing the molecular mobility of the polymer chains. $P n$-BMA has a lower $T_{\mathrm{g}}$ of $21^{\circ} \mathrm{C}$ than
Pt-BMA $\left(T_{\mathrm{g}} \sim 110^{\circ} \mathrm{C}\right)$. Then $P n$-BMA chains have a higher mobility which gave monomer molecules more efficient diffusion into nanospheres at a reaction temperature of $60^{\circ} \mathrm{C}$. Moreover, we found that broader size distributions were confirmed at both higher BMA concentrations. The reason may be that in solution, larger particles capture oligomers less efficiently due to smaller specific surface areas in total. Oligomeric radicals in solution can continue to aggregate (nucleate) to generate new nanospheres resulting in broader size distributions.

According to the data in Figure 4, the scaling relationships, which is a widely-used method able to evaluate quantitatively the effects of monomer structures on the particle size, ${ }^{25}$ obtained for $n$-BMA and $t$ BMA is as follows:

$$
R_{\mathrm{h}}=K[n-\mathrm{BMA}]_{0}{ }^{1.13 \pm 0.05}
$$

and

$$
R_{\mathrm{h}}=K[t-\mathrm{BMA}]_{0}{ }^{1.01 \pm 0.05}
$$

Exponential values in eqs 3 and 4 are significantly larger than those predicted by Winnik et al. ${ }^{18}$ who reported the gap between experimental values and theoretical values $(0.67)$. There are two reasons; 1 ) oversimplifying the theoretical model deduction process, 2) and an effect of changing BMA reaction medium concentrations; less PEG chains absorb onto particle surfaces when BMA contents are higher. Here we also calculated $n$ and $s$ values and summarized in Table II. Monomer concentration dependence of $n$ in $n$-BMA is bigger than that in $t$-BMA. On the other hand, $s$ shows no difference in monomer structures and small difference in monomer concentration.

\section{Initiator Concentration Effects}

As far as we know, there have been few publications about initiator concentration effects on particle size. ${ }^{18,26,27}$ In the present study, the initiator concentration varied from $1.8 \mathrm{mmol} / \mathrm{L}$ to $44 \mathrm{mmol} / \mathrm{L}$, while the St-PEG macromonomer and BMA concentrations

Table II. The number of grafted PEG chains on a particle surface, $n$, and the surface area occupied by one PEG chain, $s$, in the nanoparticles of PBMA- $g-P E G 2000^{\mathrm{a}}$

\begin{tabular}{cccccc}
\hline $\begin{array}{c}n \text {-BMA } \\
(\mathrm{mol} / \mathrm{L})\end{array}$ & $n \times 10^{-4}$ & $\begin{array}{c}s \\
\left(\mathrm{~nm}^{2}\right)\end{array}$ & $\begin{array}{c}t \text {-BMA } \\
(\mathrm{mol} / \mathrm{L})\end{array}$ & $n \times 10^{-4}$ & $\begin{array}{c}s \\
\left(\mathrm{~nm}^{2}\right)\end{array}$ \\
\hline 0.12 & 0.5 & 0.55 & - & - & - \\
0.24 & 3.8 & 0.45 & 0.24 & 2 & 0.55 \\
0.47 & 16 & 0.44 & 0.47 & 21 & 0.40 \\
0.93 & 33 & 0.55 & 0.93 & 31 & 0.56 \\
1.51 & 78 & 0.76 & 1.51 & 29 & 0.76 \\
- & - & - & 2.4 & 57 & 0.85 \\
\hline
\end{tabular}

${ }^{\mathrm{a}} R_{\mathrm{h}}$ values are plotted in Figure 4 and PEG2000 concentration is $10 \mathrm{mM}$. 


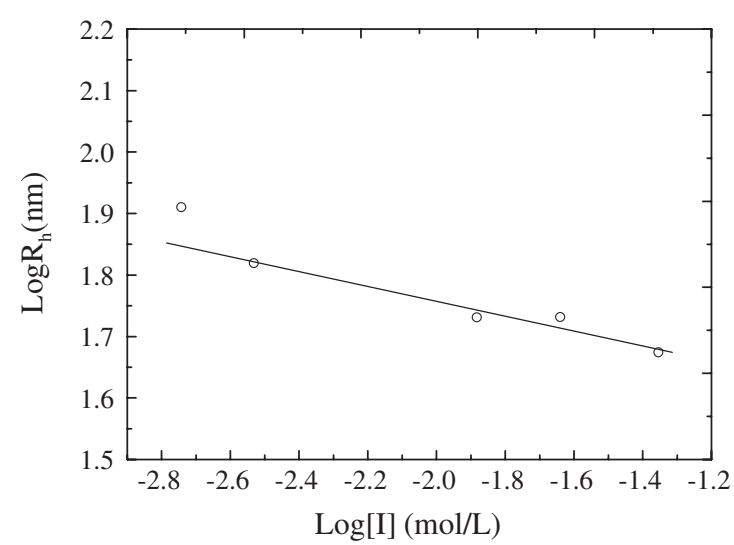

Figure 5. Double-logarithmic plot of nanosphere radii $\left(R_{\mathrm{h}}\right) v s$. [I] $[\mathrm{St}-\mathrm{PEG}]=9.26 \mathrm{mmol} / \mathrm{L},[\mathrm{BMA}]=0.463 \mathrm{~mol} / \mathrm{L}$ and $T=$ $60^{\circ} \mathrm{C}$. The solvent is ethanol containing $30 \mathrm{vol} \%$ water.

were fixed at 9.26 and $0.463 \mathrm{~mol} / \mathrm{L}$, respectively. The particle size decreases with an increase in initiator concentration, as shown in Figure 5. We obtained the following scaling relationship:

$$
R_{\mathrm{h}}=K[\mathrm{I}]_{0}{ }^{-0.12 \pm 0.01}
$$

The exponent has a good consistency to the values predicted in Paine's full adsorption model $(-0.08)^{26}$ and Winnik's model $(-1 / 12) .{ }^{18}$ A similar tendency was also observed by Chen ${ }^{6}$ and Winnik et al. ${ }^{18} \mathrm{In}$ contrast to these results, however, Paine, ${ }^{27}$ Chen et $a .^{28}$ and Tseng $^{29}$ reported that larger final particles were obtained using higher initiator concentrations in polar solvent St polymerizations. This may imply a fundamental difference in nucleation mechanisms between smaller ${ }^{6,18,26}$ and larger particles. ${ }^{27-29} \mathrm{It}$ is noticeable that particles in dispersion copolymerization of amphiphilic macromonomers simultaneously act as monomers and stabilizers in the former system. However, particles are obtained by monomer dispersion polymerization in the present study and were used as linear polymer stabilizers in the latter. Tseng et al. reported that nucleation is controlled by the adsorption or polymerization rate of stabilizer or macromonomer upon nuclei surfaces. ${ }^{29}$ In the former system, when nucleation occurs, macromonomer chains can rapidly prevent nuclei from aggregating to one another. Thus, feeding more initiator leads to the generation of more oligomeric radicals, more nuclei at nucleation, and smaller particles are obtained. However, in the latter system, the nuclei formed at nucleation are mainly stabilized by absorption to the linear polymer.

\section{Solvent Composition Effects}

It is well-known that a reaction solvent plays a key role in final particle size for dispersion polymerization. Figure 6 shows the effects of water content in

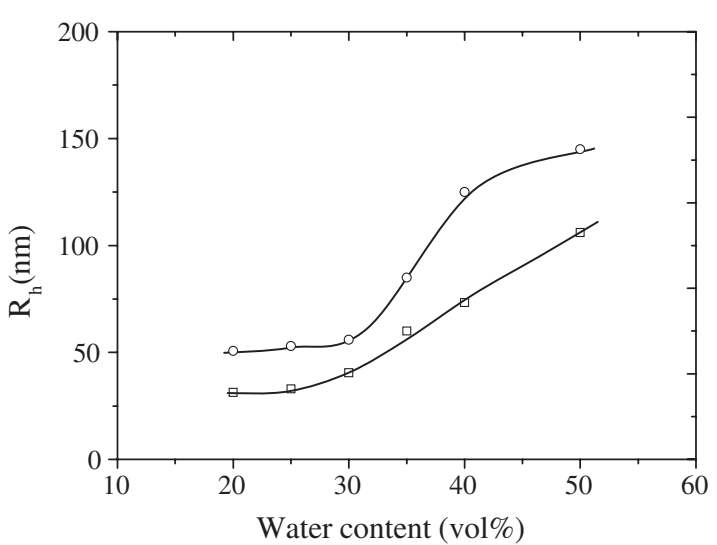

Figure 6. Solvent property effects on nanosphere $R_{\mathrm{h}}$. [StPEG $]=10 \mathrm{mmol} / \mathrm{L},[\mathrm{I}]=1 \mathrm{~mol} \%$ total monomer $(\square)$ BMA/ St-PEG $(\mathrm{mol} / \mathrm{mol})=25 ;(\bigcirc):$ BMA $/$ St-PEG $(\mathrm{mol} / \mathrm{mol})=50$.

the reaction medium on $R_{\mathrm{h}}$ of resulting nanospheres. The particle size increased with increasing water content; at the same time, the size distribution remained narrow until the water content was $50 \mathrm{vol} \%$, above which, precipitates or gels appeared. The phenomenon that final particle radii is inversely proportional to medium solubility parameters was reported by Lok and Ober ${ }^{30,31}$ In contrast, Paine ${ }^{32}$ reported that final particle size reaches a maximum when the Hansen polarity and the hydrogen term in solubility parameters are close to those of the steric stabilizers. Winnik et $a l .{ }^{18}$ found that particle sizes increased with increasing reaction medium water contents. Contrarily, Chen et al. showed a decrease in nanospheric diameters with an increased water content in the copolymerization of PNIPAAm macromonomer with $\mathrm{St}^{33}$ Thus we failed to find a general rule for reaction medium composition effects on the final particle size in dispersion copolymerization systems, since there are too many factors which complicatedly interact with one another, including solvent component interactions, solventpolymers, and different polymer chains. However, we could claim that the water content is a very significant factor to determine the particle size in BMA/StPEG systems and the poorer solvent yielded the larger particles.

\section{Solvent Sensitivity}

Figure 7 showed the effect of dialysis on nanosphere $R_{\mathrm{h}}$. After dialysis, $R_{\mathrm{h}}$ decreased to almost half of that measured prior to dialysis and the distribution of particle size also narrowed, presumably because the morphological defects of the solid nanoparticles formed during the polymerization may be dissolved under the swollen state. One explanation for the change in size is because of a change in the dispersion medium caused by dialysis. Here nanosphere cores can swell in reaction media, unreacted monomers 


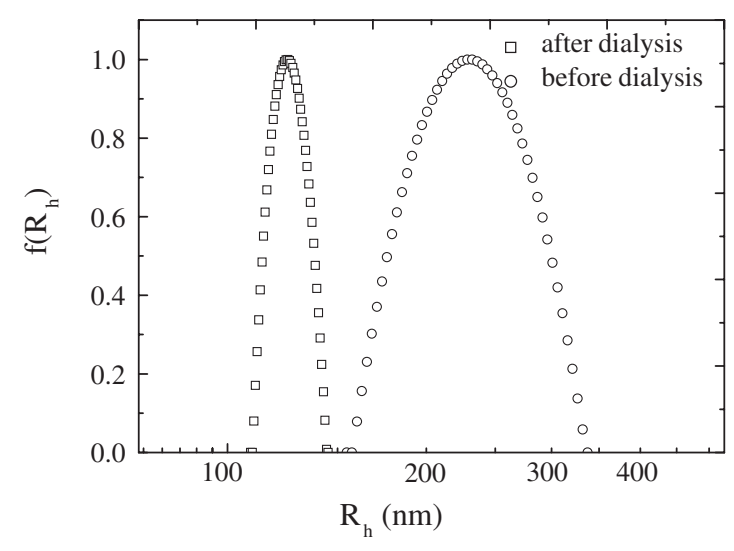

Figure 7. Hydrodynamic nanosphere radius distributions $f\left(R_{\mathrm{h}}\right)$ measured before and after dialysis against doubly distilled water. Polymerization conditions are similar to Figure 1.

and macromonomers during the reaction. However, mixed solvent and unreacted monomers are removed through dialysis against distilled water causing decreases in the degree of PBMA stretching and decreased nanospheres.

As far as we know, there is no report on non-micellar or non-crosslinked nanospheres, which can swell in certain dispersion media without destroying nanosphere morphology. Due to the solubility of PEG decreasing with the addition of ethanol in aqueous media, it is impossible to think that the PEG shell mainly contributed to morphology retention. Retention must have been caused by PBMA properties; PBMA has a low glass transition temperature $\left(T_{\mathrm{g}} \sim 21\right)$. When compared to PSt and PMMA nanospheres, PBMA chain movements are easier, and benefit solvent molecules diffusing into nanospheres, at room temperature. Thus PBMA can swell because of dispersion media. To further investigate this phenomenon, we dispersed nanospheres obtained in various media with different water/ethanol ratios and measured their sizes.

Figure 8 shows the $R_{\mathrm{h}}$ of nanospheres that were dispersed in ethanol-water mixed solvents with various mixture compositions. It is interesting to note that nanosphere sizes tend to increase with increased ethanol volumes in dispersion medium solvents. $R_{\mathrm{h}}$ did not, however, increase linearly with increased ethanol. Surprisingly, size reached a maximum at ethanol contents between 40 and $50 \%$. We are unsure of the reason for the presence of maximum particle sizes. We also measured the viscosity of PEG in ethanol/water solvents with various compositions used in preparation and found that the reduced viscosity showed a maximum at EtOH content of 50\% indicating the high solvent affinity. This solvent affinity may be one reason for showing the $R_{\mathrm{h}}$ maximum. From Figure 8 , we also find that nanosphere swelling ratios obtained by (a)

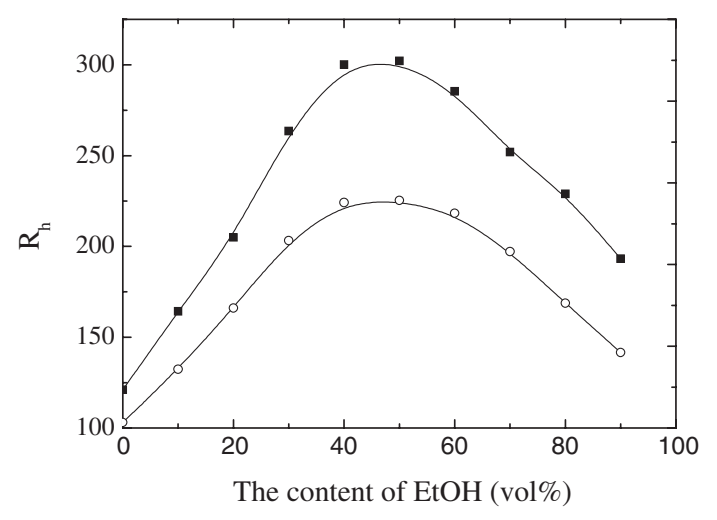

(b)

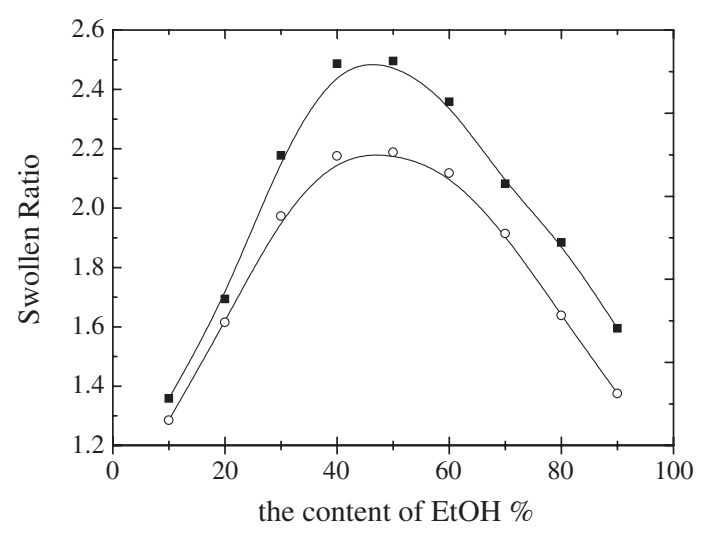

Figure 8. Dispersion medium composition effects on nanosphere $R_{\mathrm{h}}$ (a) and nanosphere swelling ratios (b). Nanospheres were obtained by copolymerization BMA $(4.6 \mathrm{mmol})$ and StPEG $(0.05 \mathrm{mmol})\left((\square): M_{\mathrm{n}}=2000,(\bigcirc): M_{\mathrm{n}}=5000\right)$ in the presence of AIBN ( $1 \mathrm{~mol} \%$ to total monomer) in ethanol/water (v/v, $7 / 3,5 \mathrm{~mL})$ at $60^{\circ} \mathrm{C}$ with a $24 \mathrm{~h}$ reaction time.

St-PEG2000 are slightly greater than those obtained by St-PEG5000. St-PEG2000 nanospheres have larger particle sizes and should have smaller coronas, which easily make us imagine larger cores. The swelling phenomenon may be directly associated with "in solvent" core swelling. Thus nanospheres comprised of PBMA-g-PEG showed core sensitivity to solvent composition and showed a volume change leading to the development of material-releasing devices with excellent colloidal dispersibility.

\section{CONCLUSIONS}

This study examined several preparation variable effects on particle size in the dispersion copolymerization of BMA with PEG macromonomers with $p$ vinylbenzyl end groups as steric stabilizers in various ethanol/water mixed solvents. Smaller particles were obtained when higher molecular weight St-PEG macromonomers were used. Monodisperse or nearly mon- 
odisperse PEG- $g$-PMBA nanospheres, ranging form ca. 40 to $750 \mathrm{~nm}$ in diameter, could be freely obtained by changing reaction parameters. Hydrodynamic radii of nanospheres $R_{\mathrm{h}}$ follow a power law dependence upon individual initial reactant system concentrations, $R_{\mathrm{h}}=K[\mathrm{I}]^{-0.12 \pm 0.01}$ [Macromonomer] $^{-0.65 \pm 0.03}$ $[\mathrm{BMA}]^{1.13 \pm 0.05}$. Reaction factor effects impact the size of resulting nanospheres and can mainly be attributed to: (1) the stabilizing ability of macromonomers; (2) the initial monomer initiator and macromonomer concentrations; and (3) polymerization process solubility parameters. It was found that resulting nanospheres could change size corresponding to changes in the ethanol/water dispersion medium compositions and swelling ratios reached a maximum at ethanol contents between 40 and $50 \mathrm{vol} \%$.

Acknowledgment. This work was financially supported by Foundation for University Key Teacher by the Ministry of Education, P. R. China (2000-65), National Science Foundation of China 50443012, and Sekisui Integrated Research.

\section{REFERENCES}

1. P. Couvreur, C. Dubernet, and F. Puisieux, Eur. J. Pharm. Biopharm., 41, 2 (1995).

2. D. Horn and J. Rieger, Angew. Chem., Int. Ed., 40, 4330 (2001).

3. M. Trau, D. A. Saville, and I. A. Aksay, Science, 272, 706 (1996).

4. A. Blaaderen, R. Ruel, and P. Wiltzius, Nature, 385, 321 (1997).

5. M. Q. Chen, A. Kishida, and M. Akashi, J. Polym. Sci., Part A: Polym. Chem., 34, 2213 (1996).

6. M. Q. Chen, T. Serizawa, A. Kishida, and M. Akashi, J. Polym. Sci., Part A: Polym. Chem., 37, 2155 (1999).

7. T. Serizawa, M. Q. Chen, and M. Akashi, Langmuir, 14, 1278 (1998).

8. M. Q. Chen, A. Kishida, T. Serizawa, and M. Akashi, J. Polym. Sci., Part A: Polym. Chem., 38, 1811 (2000).

9. M. Q. Chen, X. Y. Liu, C. Yang, and M. Akashi, Chem. Res. Chin. Univ., 18, 61 (2002).

10. T. Serizawa, M. Q. Chen, and A. Akashi, J. Polym. Sci., Part A: Polym. Chem., 36, 2581 (1998).
11. K. L. Prime and G. M. Whitesides, J. Am. Chem. Soc., 115, 10714 (1993).

12. C. Delgado, G. E. Francis, and D. Fisher, Crit. Rev. Ther. Drug Carrier Syst., 9, 249 (1992).

13. P. A. Mabrouk, Bioconjugate Chem., 5, 236 (1994).

14. A. Qin, M. Tian, C. Ramireddy, S. E. Webber, and P. Munk, Macromolecules, 27, 120 (1994).

15. F. Kohori, M. Yokoyama, K. Sakai, and T. Okano, J. Controlled Release, 78, 155 (2002).

16. J. Brandrup and E. H. Immergut, Ed., "Polymer Handbook, 3rd ed.," John Wiley \& Sons, New York, N.Y., 1989, p 382.

17. J. E. Chung, M. Yokoyama, M. Yamato, T. Aoyagi, Y. Sakurai, and T. Okano, J. Controlled Release, 62, 115 (1999).

18. S. Kawaguchi, M. A. Winnik, and K. Ito, Macromolecules, 28, 1159 (1995).

19. R. M. Fitch and C.-H. Tsai, J. Polym. Sci., Polym. Lett. Ed., 8, 703 (1970).

20. M. Akashi, T. Yamagi, E. Yashima, and N. Miyauchi, J. Polym. Sci., Polym. Chem. Ed., 27, 3521 (1989).

21. T. Kaneko, K. Hamada, M. Q. Chen, and M. Akashi, Macromolecules, 37, 501 (2004).

22. R. H. Ottewill and R. Satgurunathan, Colloid Polym. Sci., 265, 845 (1987).

23. S. Kawaguchi, A. Yekta, J. Duhamel, and M. A. Winnik, J. Phys. Chem., 98, 7891 (1994).

24. M. Riza, S. Tokura, M. Iwasaki, E. Yashima, A. Kishida, and M. Akashi, J. Polym. Sci., Part A: Polym. Chem., 33, 1219 (1995).

25. C. Wu, M. Akashi, and M. Q. Chen, Macromolecules, 30, 2187 (1997).

26. A. J. Paine, Macromolecules, 23, 3109 (1990).

27. A. J. Paine, W. Luymes, and J. Mcnulty, Macromolecules, 23, 3104 (1990).

28. Y. Chen and H. W. Yang, J. Polym. Sci., Part A: Polym. Chem., 30, 2765 (1992).

29. C. M. Tseng, Y. Y. Lu, M. S. El-Aasser, and J. W. Vanderhoff, J. Polym. Sci., Part A: Polym. Chem., 24, 2995 (1986).

30. K. P. Lok and C. K. Ober, Can. J. Chem., 63, 209 (1985).

31. C. K. Ober, K. P. Lok, and M. L. Hair, J. Polym. Sci., Polym. Lett. Ed., 23, 103 (1985).

32. A. J. Paine, J. Polym. Sci., Part A: Polym. Chem., 28, 2485 (1990).

33. M. Q. Chen, T. Serizawa, and M. Akashi, Polym. Adv. Technol., 10, 120 (1999). 